\title{
Digital Signage im stationären Handel - Das Anwendungs- und Wirkungspotenzial eines neuen POS-Mediums
}

\author{
Günter A. Silberer
}

Eingegangen: 31. August 2009 / Angenommen: 7. April 2010 / Online veröffentlicht: 22. April 2010 (C) Die Autoren 2010

Zusammenfassung Viele Kaufentscheidungen fallen am Point-of-Sale. Dies unterstreicht die Bedeutung der Kommunikation am POS. Ein neues Medium im stationären Handel nennt sich Digital Signage und steht für den vernetzten Einsatz von Flachbildschirmen. Im vorgelegten Beitrag werden die konstituierenden Merkmale sowie das Anwendungs- und Wirkungspotenzial der DigitalSignage-Technik im stationären Handel näher betrachtet. Beim Anwendungspotenzial richtet sich die Analyse auf verschiedene Funktionalitäten - auf die Verkaufsförderung, die Imageförderung, den Informationsservice und die Unterstützung der Ladenatmosphäre. Die Abschätzung des Wirkungspotenzials, die diese Funktionalitäten berücksichtigt, basiert auf einem elementaren Wirkungsmodell, auf diversen verhaltenswissenschaftlichen Ansätzen und auf Befunden empirischer Studien, die bei ersten Anwendungen in verschiedenen Ländern durchgeführt worden sind. Dabei zeigt sich ein recht breites, mehr oder weniger begrenztes Wirkungspotential der DS-Technik im Handel. Abschließend wird aufgezeigt, welche Herausforderungen sich dem heutigen Kenntnisstand zufolge für die Praxis und für die Forschung ergeben.

Schlüsselwörter POS-Medien · Store-TV · Digital Signage · In-Store Advertising · In-Store Behaviour . Shopping Behaviour

\begin{abstract}
Many buying decisions of consumers are made at the point-of-sale. This underscores the importance of pointof-sale communication and the use of in-store media. A new media in stores, shops, and buying centers is called "digital signage". This signage stands for a networked application of flat screens. The article presented here intends to show first the broad range of possible functionalities including sales promotion, image promotion, pure information service, and promoting store atmosphere. The second intention is to analyze the broad potential of impact of this new media used in retailing stores. This analysis is grounded on an elementary causal model, on different behavioral approaches, and on empirical research conducted in various countries. It is also based on a broad view concerning the functionality of digital signage. Taking a rich scientific background the broad range of possibilities to apply digital signage in the future and the rich potential of benefits of future applications are shown. Future challenges for the point-of-sale marketing of producers and retailers and implications for future research are also shown at the end of this article.
\end{abstract}

Keywords POS media - Store TV · Digital signage . In-store advertising $\cdot$ In-store behaviour · Shopping behaviour

\section{Einleitung}

Viele Kaufentscheidungen fallen erst am Point-of-Sale (Stottmeister 1990; Riediger u. Röder 2007; OgilvyAction 2007). Besucher tätigen Käufe, die gar nicht vorgesehen waren. Und selbst dann, wenn ein Besucher mit einer Kaufabsicht das Geschäft betritt, kann es sein, dass die Entscheidung darüber, was genau in welcher Menge gekauft

\author{
G. A. Silberer (ه) \\ Georg-August-Universität Göttingen, \\ Institut für Marketing und Handel, \\ Platz der Göttinger Sieben 3, 37073 Göttingen, Deutschland \\ E-Mail: silberer@guentersilberer.de \\ Tel.: +49-551-43092 \\ URL: guentersilberer.de
}


werden soll, erst vor Ort getroffen wird. Diese Sachverhalte erklären und unterstreichen die Bedeutung des Point-of-Sale (POS) für den Absatzerfolg von Herstellern und Händlern (Burke 2006, 2009). Um Absatzerfolge am POS zu steigern und zu sichern, treiben Hersteller und Händler POS-Marketing. Dabei werden attraktive Ladenumwelten geschaffen, Angebote ansprechend präsentiert, Preise kommuniziert und auch Medien eingesetzt. Als klassische POS-Medien kennen wir im stationären Handel Innenplakate, Regalbeschriftungen, Deckenhänger, Displays, Musik und Durchsagen (Barth et al. 2002, S. 242-244; Schnedlitz u. Berger 2005, S. 305). Zu den POS-Medien zählt aber auch das Ladenfernsehen, das In-Store TV. Der Einsatz von Medien im stationären Handel erklärt sich vor allem daraus, dass selbst gute Regalplatzierungen nicht immer ausreichen, um die erwünschte Aufmerksamkeit zu erzielen (Chandon et al. 2009).

Das Ladenfernsehen hat in den vergangenen Jahren eine weitere Entwicklungsstufe erreicht. Die erste Stufe, die vor Jahren zu Ende ging, bestand aus Röhrengeräten, die wie Zimmergeräte als Insellösungen im Laden verteilt waren und audiovisuelle lineare Inhalte per Endlosschleife darboten. Mit der digitalen Revolution kamen vor etwa fünfzehn Jahren in einer zweiten Stufe die interaktiven Multimedia-Terminals mit dem anwählbaren (Hyper-)Text, der auch audiovisuelle Darstellungen beinhalten konnte, zum Einsatz (Swoboda 1996, 1998; Silberer 1999, 2000; Silberer u. Fischer 2000; Silberer et al. 2004; EHI 2005; Liebmann et al. 2008, S. 592). Die Geräte in diesen beiden Entwicklungsstufen konnten allerdings keine nennenswerte Verbreitung erringen. Während die Röhrengeräte oft mit der Größe der Bildschirme und lieblos gestalteten Inhalten zu kämpfen hatten, wurde die Terminal-Lösung als stationäre Multimedia-Anwendung vom hohen Investitionsbedarf, vom aufwendigen Pflegeaufwand und von der geringen Bereitschaft der Ladenbesucher gebremst, sich beim Einkaufen via Terminal mit Informationen und Werbebotschaften zu versorgen. Mit den hochauflösenden Flachbildschirmen in vernetzten und zentral gesteuerten Systemen, dem Digital Signage $(D S)$, befinden wir uns derzeit zu Beginn der dritten Entwicklungsstufe des Store-TV. Mit dem DS dürfte es gelingen, jene Hemmnisse zu überwinden, mit denen die Vorgängersysteme zu kämpfen hatten. Die Prägnanz der Darstellung, die Größe und Reichweite der Bildschirme, deren Steuerbarkeit durch die Zentrale und die Tatsache, dass die Ladenbesucher keine Inhalte aufrufen müssen, aber auch der Preisverfall bei der erforderlichen Hardware und Software sprechen für eine solche Einschätzung (IfH-Studie 2007; Burke 2009; Gerba 2009; Todd 2009; Van Loock et al. 2009).

Der vernetzte Einsatz von Flachbildschirmen im stationären Handel konnte in der Fachliteratur bislang keine große Beachtung finden. Bei den ersten Darstellungen, die in erster Linie von Dienstleistern stammen und oft der Eigenwerbung dienen, ist festzustellen, dass DS - wenn überhaupt - nur vage umschrieben und in seinen konstituierenden Merkmalen nur lückenhaft spezifiziert wird (Sörensen 2009; www.black-box.de; www.ekiosk.de). Und bei der beschriebenen Funktionalität steht die Verkaufsförderung nach wie vor im Mittelpunkt.

Wenn sich das DS im stationären Handel als ein Instrument der Verkaufsförderung anbietet, so ist damit das Potenzial dieser Technik keineswegs erschöpft. Deshalb soll in diesem Beitrag das gesamte Anwendungspotenzial der DS-Technik herausgestellt werden. Dies dient nicht nur der besseren Beurteilung der Einsatzmöglichkeiten, sondern auch der besseren Abschätzung des Wirkungspotenzials. Diese Beurteilung ist nicht nur für die Wissenschaft, sondern auch für die Praxis eine große Herausforderung. Deshalb wird in diesem Beitrag auch der Versuch unternommen, das Wirkungspotenzial der DS-Technik zu skizzieren. Dabei kann nicht nur auf grundlegende Erkenntnisse der Medien-, Werbewirkungs- und Käuferforschung zurückgegriffen werden, sondern auch auf erste Studien, die sich mit Wirkungen des DS am POS befassen. Auf der Grundlage einer näheren Betrachtung unterschiedlicher Funktionalitäten und entsprechender Wirkungspotenziale sei schließlich der Frage nachgegangen, worauf Forschung und Praxis künftig ihr Augenmerk legen sollten und welche Folgerungen dabei aus den bisherigen Erkenntnissen zu ziehen sind.

\section{Die konstituierenden Merkmale des Digital Signage}

Digital Signage bezeichnet einer gängigen Definition folgend den vernetzten, digitalisierten Einsatz von Flachbildschirmen als Informations- und Werbeplattform (Burke 2009; Sörensen 2009; Van Loock et al. 2009). Etwas näher betrachtet besteht ein DS-System aus einem Server oder mehreren Servern, die digitale Inhalte bereitstellen, und von den Clients, die diese Inhalte abrufen können. Die abrufenden Rechner bedienen die einzelnen Bildschirme vor Ort - in der Regel Plasma- bzw. LCD-Bildschirme. Übertragungswege können Kabelnetze, Mobilfunk- und Satellitennetze sein. Auf der Seite der Produktion und $\mathrm{Zu}-$ lieferung von Inhalten sind ebenfalls Rechnernetze denkbar, die an unterschiedlichen Orten Inhalte aufbereiten und von diesen Orten die Inhalte zuspielen. Denkbar sind auch Sensoriksysteme, welche die Präsentation von Inhalten steuern, so z.B. Bewegungsmelder, die ankommende Besucher signalisieren, oder Scannerkassen, die den Abverkauf, ggf. auch den Ausverkauf umworbener Produkte melden. $\mathrm{Zu}$ den Software-Komponenten gehören die Übertragungssoftware und die Präsentationssoftware - meist gängige TCP/IP Programme. 
Ein DS-System kann im stationären Handel für einzelne Ladengeschäfte, aber auch für Filialketten und ganze Einkaufszentren eingerichtet sein. Als Bildschirmstandorte kommen Eingangsbereiche, Ausgangs- oder Kassenbereiche, Bereiche der Warenpräsentation, Servicestationen und Restaurants, aber auch Schaufenster und Außenwände in Frage. Bildschirme können an Wänden, Pfeilern, in Regalen und anderen großflächigen Warenträgern angebracht sein. Über die Reichweite einzelner Bildschirme entscheiden neben der Bildschirmgröße und der Aufhängungshöhe räumliche Gegebenheiten wie z. B. die Raumaufteilung, die Möblierung und die Wegeführung. Auf den Bildschirmen lassen sich statische Inhalte wie Bilder, Logos, Slogans und kurze Texte ebenso darstellen wie dynamische Inhalte, so z. B. Videos, Animationen und Laufschriften. Der Einsatz von Sound bzw. die Wiedergabe von gesprochenem Wort, von Jingles und von (Hintergrund-)Musik ist ebenfalls denkbar.

Das Neue und Nützliche der DS-Systeme besteht vor allem in der Möglichkeit, auf raumsparenden hochauflösenden Flachbildschirmen Inhalte jeder Art multimodal darzubieten, diese Inhalte zentral und dezentral zu steuern und auf Filialen, Abteilungen und Standorte gezielt auszurichten. Im Gegensatz zum „Paper Signage“ lassen sich die Inhalte jederzeit und ohne großen Aufwand ändern. Wichtig ist auch der Umstand, dass aktuelle Informationen ohne zeitliche Verzögerung angeboten werden können. Wenn DS gelegentlich wortwörtlich mit „Digitale Beschilderung“ übersetzt wird, mag diese Übersetzung zwar korrekt sein, kann dem Potenzial der DS-Systeme jedoch nicht gerecht werden.

\section{Anwendungspotenzial und -felder des Digital Signage}

Das Anwendungspotenzial

Das Anwendungspotenzial des DS lässt sich über die möglichen, unterschiedlichen Funktionalitäten verdeutlichen. Neben der Verkaufsförderung kommen vor allem die Imageförderung, die Informationsfunktion und die Förderung der Ladenatmosphäre in Betracht (vgl. Liebmann et al. 2008, S. 612). Diese vier Funktionen werden im Folgenden näher betrachtet. $\mathrm{Zu}$ weiteren Aufgaben, die in diesem Beitrag nicht näher behandelt werden können, zählen Werbewirkungstests sowie die Förderung der Corporate Identity.

Verkaufsförderung bedeutet vor allem, die Nachfrageseite zum Kauf von Angeboten anzuregen. Bezogen auf die Ladenbesucher bedeutet dies, den Kauf von Produkten vor Ort zu fördern. Dazu zählen das Auslösen ungeplanter Käufe und die Steigerung von Stückzahlen bei Produkten, deren Kauf ohnehin beabsichtigt war. Diese Verkaufsförderung kann vom Händler ausgehen, der z. B. auf zeitlich begrenzte Preisreduktionen und/oder auf begrenzte Angebotsmengen hinweisen möchte, aber auch von den Herstellern, die durch günstige Konditionen die Reduktion des Endverkaufspreises ermöglichen, POS-Werbung unterstützen und eigenes Personal für den Einsatz am POS zur Verfügung stellen. Beide Seiten können per DS ausgestrahlte Botschaften die Abverkäufe am POS unterstützen.

Wie die Imagewerbung in anderen Kanälen können Inhalte im DS-System auch darauf abzielen, die Einstellungen gegenüber Anbietern und ihren Angeboten zu verbessern. Dabei geht es um die Steuerung von Vorstellungen und Emotionen, die mit Anbietern und Angeboten verbunden sind. Einzelne Handelsbetriebe und ganze Handelsketten können versuchen, mit dem Einsatz von DS ihr Image und das ihrer Angebote zu fördern (Brown 1974; Sattler u. Hartmann 2004, S. 12). Dabei kommen vor allem bildkräftige und emotional aufgeladene Inhalte sowie deren wiederholte Darbietung in Frage. Der Umstand, dass bei großen Bildschirmen plakative und bunte Darstellungen möglich sind, kommt einer Imageförderung durchaus entgegen. Für eine solche Nutzung des DS können unter anderem der „Image-Malus des Handels“ (Mattmüller 1999, S. 391 f.), das Bemühen um ein erfolgreiches „Retail Branding“ (Morschett 2006, S. 532 f.) oder die Erkenntnis sprechen, dass ein permanentes „Discounting“, das sich in wiederholten Preissenkungen oder in Dauerniedrigpreisen des Handels niederschlägt, auf die Dauer einen „Irrweg“ darstellt (Diller 1999, S. 356-358; Fritz et al. 2007). Die Alternative dazu wäre ein verstärktes Bemühen um ein vorteilhaftes Sortiments- und Produktimage. Kozinets (2008, S. 8-10) spricht hier von einer Entwicklung der Einzelhandelsgeschäfte zu ,brand spaces and brand places“. Bei solchen Zielen kommt auch der Einsatz des DS in Frage, die mediale Imagepflege vor Ort.

Ein DS-System kann aber auch so ausgelegt werden, dass die Information der Besucher bzw. der Informationsservice im Vordergrund steht. Dabei ist nicht nur an Slogans und Texte $\mathrm{zu}$ denken, sondern auch an Abbildungen, Videoclips und Animationen. Als Informationsdienste kommen im stationären Handel unter anderem aktuelle Wetterdienste, Hinweise auf Veranstaltungen in der Region, Verweise auf eigene Kundenzeitschriften und Websites, auf Auslieferungs-, Verpackungs- und Rücknahmedienste sowie Zubereitungs-, Lagerungs- und Installationstipps in Frage. Im Zeitalter der breiten und tiefen Sortimente und der Verkaufsflächenexpansion im stationären Handel (Lademann 1999, S. 530-536) können Informationssysteme auch einen Sortimentsüberblick liefern und so der „Konsumentenverwirrtheit“ (Walsh 2002) entgegenwirken. Denkbar sind - analog zum „Web 2.0“ - selbst Ratschläge anderer Kunden (zu „,sozialen Medien“ mit kundenseitig generierten Inhalten im Handel (siehe Constantinides et al. 
Tabelle 1 Bedeutsame DS-Funktionalitäten in unterschiedlichen Betriebstypen

\begin{tabular}{lllll}
\hline & $\begin{array}{l}\text { Verkaufs- } \\
\text { förderung }\end{array}$ & $\begin{array}{l}\text { Image- } \\
\text { förderung }\end{array}$ & $\begin{array}{l}\text { Informations- } \\
\text { service }\end{array}$ & $\begin{array}{l}\text { Laden- } \\
\text { atmosphäre }\end{array}$ \\
\hline Flag Ship Stores & - & $\mathbf{x}$ & - & $\mathbf{x}$ \\
Kaufhäuser & $\mathbf{x}$ & $\mathbf{x}$ & - & $\mathbf{x}$ \\
Supermärkte & $\mathbf{x}$ & - & - & - \\
Convenience Stores & $\mathbf{x}$ & - & $\mathbf{x}$ & - \\
Fachgeschäfte & $\mathbf{x}$ & - & $\mathbf{x}$ & - \\
\hline
\end{tabular}

2008). Schließlich kommen auch neutrale Verbraucherinformationen in Frage, z. B. Ergebnisse vergleichender Waren- und Dienstleistungstests sowie Hinweise auf die Aussagekraft von Gütesiegeln und Qualitäts- und Umweltzeichen. Mit einem solchen Angebot können Händler einen wertvollen Kundendienst leisten und damit ihre „Informationskompetenz“ (Rudolph 2005, S.176 f.) unter Beweis stellen.

DS kann auch die Aufgabe übernehmen, atmosphärische Wirkungen $\mathrm{zu}$ erzeugen und so die Ladenatmosphäre positiv beeinflussen. Diese Funktionalität liegt insofern nahe, als die Ladenatmosphäre in einer Zeit des Erlebniseinkaufs an Bedeutung gewonnen hat (Bost 1987, S. 30-56; Gröppel 1991, S. 32-41; Gröppel-Klein 2006, S. 678 f.; Newman et al. 2006, S. 3 f.; Ahlert u. Kenning 2007, S. 278 f.; Liebmann et al. 2008, S. 606-610; Dennis et al. 2008, S. 5; Schweiger u. Schrattenecker 2009, S. 120; Kroeber-Riel et al. 2009, S. 612-614). Wenn das Einkaufen möglichst angenehm und abwechslungsreich erlebt werden soll, kann DS am POS als ,ambient factor“ an verschiedenen Punkten ansetzen. Einen ersten Ansatzpunkt liefert die Ladenästhetik. Ansprechende Darstellungen auf großen hochauflösenden Screens mit passenden Farben und Bildern sind in der Lage, wie Kunstwerke oder wie Wandschmuck zu wirken, Wandflächen aufzuwerten und die Anmutung eines ,Warenlagers“ abzubauen (vgl. Kroeber-Riel 1993; Jacobsen 1994; Silberer et al. 2000, S. 151-160). Einen zweiten Ansatzpunkt bietet die Unterhaltung, so z. B. die von Schüppenhauer (2000, S. 418) erwähnten kleinen „Erzählungen aus der Alltagswelt“. Ein dritter Ansatzpunkt bezieht sich auf die soziale Dimension der Ladenatmosphäre. Bildschirme im Eingangsbereich können Besucher freundlich begrüßen und Bildschirme im Ausgangsbereich freundlich verabschieden (Silberer 2009). Außerdem lassen sich Mitarbeiter vorstellen, und zwar einzelne Mitarbeiter ebenso wie ganze Teams.

Die Anwendungsfelder

Welche Funktionalität sich beim Einsatz und bei der Ausgestaltung eines DS-Systems anbietet, hängt unter anderem vom jeweiligen Einsatzgebiet und damit auch von der Betriebsform und der jeweiligen Zielgruppe ab. Flag-Ship
Stores, Kaufhäuser, Supermärkte, Convenience Stores und Fachgeschäfte eröffnen unterschiedliche Möglichkeiten und verlangen unterschiedlich ausgelegte DS-Systeme. Die Verkaufsförderungsfunktion kann in allen Betriebstypen des Handels von Bedeutung sein, verglichen mit Supermärkten und Discountern ist deren Bedeutung in Flag-Ship Stores eher etwas geringer. Dafür bietet sich in Flag-Ship Stores in erster Linie die Imagefunktion an. Hier kann ein Haus oder eine Kette wichtige Anregungen in Richtung Life-Style und Konsummuster geben. Ein DS-basierter Informationsservice macht vor allem in Fachgeschäften Sinn, kommt aber auch als Nachrichtensystem in Convenience Stores auf Autobahn-Raststätten in Frage. In solchen Stätten wäre aber auch an touristische Informationsdienste $\mathrm{zu}$ denken, so z. B. gezielte Wettervorhersagen sowie Hinweise auf Restaurants, Hotels und Ferienwohnungen. Die besondere Bedeutung der atmosphärischen Funktion eines DS-Systems ergibt sich schließlich für jene Betriebstypen, die auf ein angenehmes Shoppingerleben, auf Verweilen und auf Life Style-bezogene Anregungen setzen. Wenn solche Tendenzaussagen recht allgemein gehalten sind, so liegt dies daran, dass die konkrete Funktionalität einer DS-Anwendung im Einzelfall entschieden und an den jeweiligen Zielen und Zielgruppen ausgerichtet werden muss. Dies gilt letztlich auch für die Interpretation der in Tabelle 1 gemachten Angaben.

\section{Das Wirkungspotenzial des Digital Signage}

Nach den möglichen Funktionalitäten und Ausformungen eines DS-Systems im stationären Handel interessiert nun deren Wirkungs- und Nutzenpotenzial. Nach der Skizze eines elementaren Wirkungsmodells, das als ein theoretischer Bezugsrahmen dient, folgt die nähere Betrachtung des Wirkungs- und Nutzenpotenzials, bei der die funktionale Auslegung eines DS-Systems im Vordergrund steht.

Ein Wirkungsmodell als theoretischer Bezugsrahmen

Die Abschätzung des Wirkungspotenzials eines DSSystems geht hier von folgenden Grundannahmen aus: 1) Entscheidende Wirkungsdeterminanten sind zunächst 
Abb. 1 Ein elementares Wirkungsmodell als theoretischer Bezugsrahmen

\begin{tabular}{|c|c|c|c|c|c|c|}
\hline \multirow{2}{*}{$\begin{array}{l}\text { System- } \\
\text { merkmale } \\
\\
\text { Standorte }\end{array}$} & \multicolumn{2}{|r|}{$\begin{array}{l}\text { Kontakt- } \\
\text { erfolge }\end{array}$} & \multicolumn{2}{|c|}{$\begin{array}{l}\text { psychische } \\
\text { Wirkungen }\end{array}$} & \multicolumn{2}{|c|}{$\begin{array}{l}\text { verhaltensbezogene } \\
\text { Wirkungen }\end{array}$} \\
\hline & $\rightarrow$ & Kontakt- & $\rightarrow$ & kognitive & $\rightarrow$ & Verweildauer \\
\hline Umfelder & $\rightarrow$ & -dauer & $\rightarrow$ & Wirkungen & $\rightarrow$ & Informations- \\
\hline Inhalte & $\rightarrow$ & -anzahl & $\rightarrow$ & affektive & $\rightarrow$ & verhalten \\
\hline \multirow[t]{2}{*}{ Timing } & $\rightarrow$ & -intensität & $\rightarrow$ & Wirkungen & $\rightarrow$ & Kaufverhalten \\
\hline & $\rightarrow$ & Ablenkung & $\rightarrow$ & & $\rightarrow$ & Empfehlungen \\
\hline
\end{tabular}

die Systemmerkmale, vor allem die Abdeckung der Ladenflächen und die Reichweite der Bildschirme, die jeweiligen Umfelder (ob z. B. an der Kasse oder im Verkaufsbereich und dort die Nähe zu beworbenen Angeboten), die dargebotenen Inhalte (z. B. der Werbe-, Info- und Unterhaltungsmix) und das Timing (Jahreszeit, Wochentag, Tageszeit). 2) Zu den unmittelbaren Erfolgswirkungen zählen die Kontakte, genauer die Sichtkontakte, ggf. auch die Wahrnehmung akustischer Inhalte wie Hintergrundmusik oder Ansagen. Diese Kontakte unterscheiden sich in ihrer Dauer, Intensität und Anzahl, bezogen auf einen einzelnen Standort oder Bildschirm und bezogen auf das Ladengeschäft oder den Ladenbesuch insgesamt. 3) Aus Kontakten bzw. Wahrnehmungserfolgen resultieren kognitive, affektive und konative Wirkungen. Zu den kognitiven Wirkungen zählen z. B. Wissenseffekte, zu den affektiven Wirkungen z. B. Emotionen und Stimmungen, zu den konativen Wirkungen z. B. die Besuchsdauer, die Informationssuche, die Kaufabsichten, das Kaufverhalten, das Konsumverhalten und die Weiterempfehlung. 4) Werden längere Zeiträume betrachtet, kommen nicht nur sog. Adoptionsprozesse (Rogers 2003), hier die regelmäßige Nutzung des POS-Mediums, sondern auch zeitlich verzögerte Kaufeffekte sowie die Ladentreue als DS-Wirkungen auf der Verhaltensebene in Betracht. Von den längerfristigen Erfolgen abgesehen werden die skizzierten Zusammenhänge in Abb. 1 ganz grob wiedergegeben.

Art und Umfang des Wirkungspotenzials einer DSAnwendung im stationären Handel hängen nicht nur vom DS-System selbst, sondern auch von der Ladenumgebung und vom Ladenbesucher selbst ab. Entscheidend sind zunächst die Einsehbarkeit und die Inhalte der per Bildschirm ausgestrahlten Botschaften. Bezüglich der Ladenumgebung kommt es vor allem auf die Angebote und Preise vor Ort, auf die Präsentation des Angebots und auf konkurrierende sowie ergänzende Informationsquellen wie z. B. auf die Präsenz von Mitarbeitern am Servicepoint und an der Fremdbedienungstheke an. Bezogen auf den Ladenbesucher selbst sind es vor allem persönliche Interessen, Einstellungen und Zahlungsbereitschaften, die das Wirkungsgeschehen beeinflussen (vgl. Titus u. Everett 1995; Zielke 2002, S. 85-89), aber auch das Lauf- und Zuwendungsverhalten, das z. T. festen Gewohnheiten folgt, aber auch von der Ladengestaltung abhängt (Gröppel-Klein u. Bartmann 2009), und darüber mit entscheidet, ob Bildschirme überhaupt in das Blickfeld der Ladenbesucher rücken. Und nicht jeder Ladenbesucher möchte sich beim Einkaufen mit medial dargebotenen Inhalten befassen, weil er die unmittelbare Betrachtung und eigenständige Beurteilung der Angebote vorzieht (vgl. Sörensen 2009, S. 176).

\section{Zum Wirkungspotenzial bei einzelnen Funktionen}

Eine nähere Betrachtung möglicher Wirkungen eines DS am POS folgt nun im Lichte diverser Funktionalitäten. Aus Platzgründen können dabei nur einige wenige Wirkungspfade und Wirkungsdeterminanten herausgestellt werden.

\section{Zum Wirkungspotenzial DS-basierter Verkaufsförderung}

Bezogen auf eine DS-basierte Sales Promotion bei Sonderangeboten und bei saisonalen Angeboten lassen sich unter anderem folgende Wirkungen annehmen:

\section{a) Zu den Kontakterfolgen}

Attraktive und schnell erfassbare Werbebotschaften im Dienst einer Sales Promotion haben gute Chancen, am POS auch wahrgenommen zu werden. Dies hängt nicht nur von den Inhalten, sondern auch davon ab, ob ein Besucher sich auf der gezielten Produktsuche befindet oder sich auch oder vor allem ein wenig umschauen, sich orientieren will. Damit geht eine recht unterschiedliche Offenheit für Reize bzw. Hinweise einher (vgl. Gröppel 1991, S. 53). Der „Seeker“ ist nur für das offen was er sucht, ggf. auch 
für mediale Botschaften vor Ort, die ihm helfen, seine Kaufpläne schnell und erfolgreich zu realisieren.

\section{b) Zu den kognitiv-affektiven Wirkungen}

Kognitiv-affektive Wirkungen hängen von der Gestaltung der Inhalte, aber auch von der Stärke und von der Ausprägung des Involvement ab. Bei geringem Involvement sind bestenfalls flüchtige Kontakte zu erwarten, bei denen sog. Hinweisreize wirksam werden, bei starkem Involvement eher eine aufmerksame Zuwendung, bei der auch Argumente zählen und bei der die vorgetragenen Argumente auch kritisch verarbeitet werden (Krugman 1965; Petty u. Cacioppo 1984, 1986; Karremans et al. 2006). Demzufolge können bei flüchtigen Werbekontakten allein schon ansprechende Produktabbildungen frühere Kaufabsichten in Erinnerung rufen sowie Kauf- oder Konsumwünsche wecken (vgl. Van Loock et al. 2009, S. 1-4). Aufmerksame Zuwendungen zu Werbebotschaften schließen dagegen selbst nachhaltige Überzeugungs- und Lerneffekte (Hawkins et al. 2001) nicht aus. Bei einem kognitiven Involvement, das auf Sachargumente setzt, können z. B. Hinweise auf die Qualität, den Preis, das Herkunftsland und die Haltbarkeit auf Interesse stoßen (vgl. Brown 1974; Woodside u. Waddle 1975), bei einem affektiven Involvement dagegen die Art der Präsentation und die Produktanmutung. Was den Besucher gerade interessiert, beeinflusst seine situative Wahrnehmung, seine „situated cognition“ (Schwarz 2002; Smith u. Semin 2004; Fischer 2002, S. 137 f.; Büttner 2009, S. 21-28). Diese Wahrnehmung hängt aber auch davon $\mathrm{ab}$, wie er sich gerade fühlt und in welcher Stimmungslage er sich gerade befindet.

\section{c) Zu den Verhaltenswirkungen}

Für die Wirksamkeit einer Sales Promotion am POS spricht vor allem der Umstand, dass die Absichten, Erwartungen und Ortskenntnisse des Ladenbesuchers in der Lage sind, die Gedanken vor Ort in Richtung Kauf und Konsum zu bahnen, und dass diese Bahnung von Gedanken von den Angeboten und Botschaften vor Ort verstärkt werden kann. Dieses sog. „Framing“ (Tversky u. Kahnemann 1981; Druckman 2001; Clark 2009), eine Art „Fit zwischen Denkstil und Situation“ (Novak u. Hoffman 2009), trägt dann auch dazu bei, dass sich Kommunikations- und Kaufbarrieren absenken und sich die Wirksamkeit von Kaufanreizen wie z. B. einer Preisreduktion verstärkt (vgl. Brown 1974, S. 36; Woodside u. Waddle 1975, S. 30; Burke 2009, S. 185; Kroeber-Riel et al. 2009, S. 448; Schweiger u. Schrattenecker 2009, S. 386 f.). Selbst einzelne Käufe können das Framing verstärken und die Wahrscheinlichkeit weiterer Käufe steigern. Dhar et al. (2007) berichten einen solchen Effekt und sprechen von einem „Shopping Momentum“, das man auch „Kaufschub“ nennen könnte. Gelingt es somit, per DS einen Kauf aus- zulösen, steigt damit zugleich die Chance, dass ein weiterer Kaufanreiz ebenfalls zum Erfolg, z. B. zu einem Impulskauf oder zu einer Steigerung der gekauften Artikelanzahl, führt (siehe dazu die Erörterung sog. „thin slice decisions“ bei Wang et al. 2008).

\section{Zum Wirkungspotenzial imagefördernder DS-Systeme}

Bei der Frage nach den Wirkungen eines DS, das auf Imageerfolge abzielt, sei vor allem solche Anwendungen abgestellt, die zentrale Botschaften über das Leistungsprogramm des Händlers und das einzelner Angebote, z. B. bestimmter Herstellermarken, an verschiedenen Stellen des Geschäfts wiederholt kommunizieren.

\section{a) $\mathrm{Zu}$ den Kontakterfolgen}

Die wiederholte Kommunikation zentraler Botschaften über das Leistungsprogramm des Händlers und über einzelne Angebote, z. B. über bestimmte Herstellermarken, an verschieden Stellen eines Geschäfts ist mit der Chance verbunden, wiederholte Kontakte auch bei solchen Besuchern zu erzielen, die den Bildschirmen und den Inhalten keine nennenswerte Aufmerksamkeit schenken. Dabei sind nicht nur mehrere ungewollte, mehr oder weniger flüchtige Kontakte zu erwarten, sondern auch gewollte oder gezielte Kontakte, wenn im Laufe der Zeit mit solchen Kontakten auch positive Erfahrungen gemacht werden konnten.

\section{b) Zu den kognitiv-affektiven Wirkungen}

Kognitiv-affektive Wirkungen, z. B. das Herausbilden von Einstellungen (das ,imagery“) und die Veränderung von Einstellungen, sind bei flüchtigen Kontakten am ehesten dann $\mathrm{zu}$ erzielen, wenn es $\mathrm{zu}$ wiederholten Kontakten kommt (Kroeber-Riel et al. 2009, S. 387). Die dabei erlernten Assoziationen und Anmutungen können nicht nur das Sortiment im Ganzen, sondern auch einzelne Marken bestimmter Hersteller betreffen.

Bezogen auf die Imagepflege im Handel, bei der der Handel sein eigenes Image fördert, sprechen Foscht u. Angerer (2006) von einer „Positionierung durch Emotion, Information und Aktualität". Dies ist gerade bei Inhalten des DS oft der Fall. Emotion kann z. B. durch ansprechende Bilder und unterhaltsame Aussagen erzeugt werden, Information durch nützliche Angaben in Sachen Qualität, Haltbarkeit, Lagerung und Zubereitung und Aktualität durch neue Testberichte sowie Hinweise auf neue Produkte und neue Qualitäts- oder Umweltzeichen.

Das besondere Potenzial einer Imageförderung am POS resultiert daraus, dass Versprechungen zum großen Teil vor Ort und ohne zeitliche Verzögerung unter Beweis gestellt bzw. überprüft werden können. Gilt es, das Händlerimage zu fördern, so kann das Leistungspotenzial mit den verschiedenen Sortimentsdimensionen unterstrichen werden - mit 
der Sortimentsstruktur, die die Aufteilung des Leistungsprogramms betrifft, mit dem Sortimentsniveau, das die Produktqualität, den Präsentationsaufwand, das Serviceniveau und das Preisniveau betrifft, mit der Sortimentsbreite, die additive Kaufmöglichkeiten eröffnet, und mit der Sortimentstiefe und damit mit den Auswahlmöglichkeiten in den einzelnen Sortimentsteilen. Und soll die Beurteilung einzelner Angebote gefördert werden, was auch über Werbebotschaften der Hersteller erfolgen kann, so können auch Verkostungen die Berechtigung werblicher Versprechungen unterstreichen.

\section{c) Zu den Verhaltenswirkungen}

Einstellungen werden nicht nur über unmittelbare Kommunikationseffekte erzielt, sondern auch mittelbar über Verhaltenswirkungen. Dies ist z. B. dort der Fall, wo über ein DS Kauf- und Konsumerfolge erzielt werden konnten und diese wiederum das Image des Händlers und der betroffenen Produkte beeinflussen. Dasselbe gilt dort, wo angebotene Serviceleistungen in Anspruch genommen wurden und in ihrer Nützlichkeit beurteilt werden können. War oben vom Assoziationslernen die Rede, so ist hier das Lernen aus Erfahrungen angesprochen, von dem besonders nachhaltige Imagewirkungen erwartet werden können.

\section{Zum Wirkungspotenzial DS-basierter Informationsdienste}

DS-basierte Informationsdienste können eine breite Palette unterschiedlicher Nachrichten, Hilfestellungen und Anregungen beinhalten, so dass sich deren Wirkungen hier nur grob skizzieren lassen.

\section{a) Zu den Kontakterfolgen}

Da sich viele Besucher im Geschäft in erster Linie dem Angebot zuwenden (Burke 2009, S. 184 f.), ist auch der Kontakt mit den Informationsdiensten keineswegs gesichert. Es gibt jedoch Stellen, an denen am ehesten mit einem gewissen Informationsinteresse gerechnet werden darf. Dies gilt unter anderem für die Übersichten am Eingang (die sog. „entrance maps“, siehe Liebmann et al. 2008, S. 614), für unmittelbar produktrelevante Hinweise im Verkaufsbereich, so z. B. Testberichte, Hinweise auf Qualitätsauszeichnungen und die Vorstellung wichtiger Lieferanten, für Zubereitungsideen im Restaurantbereich und für Hinweise auf den Lieferservice in Verpackungsstationen.

\section{b) Zu den kognitiv-affektiven Wirkungen}

Informationsdienste, die vom Händler ausgehen, kommen vor allem ihm zugute, Hinweise auf Serviceleistungen bestimmter Hersteller oder anderer Institutionen aber auch diesen Akteuren (zur „Informationskompetenz“ des Handels siehe Rudolph 2005, S. 175-190). Testberichte, die der Händler kommuniziert und die auf die gute Qualität bestimmter Produkte hinweisen, fördern ebenfalls den Ruf des Händlers und des Herstellers gleichermaßen. Hinweise, die den Suchaufwand begrenzen (Sörensen 2009), fördern zunächst die Kaufzufriedenheit, letztlich aber auch das Image des Geschäftes (Burke 2009, S. 184 f.).

Bestehen DS-Inhalte aus einer Mischung von Nachrichten, Informationsdiensten und Werbung des Händlers und der Hersteller, kann der wahrgenommene Informationswert des gesamten Bündels dazu führen, dass die integrierten Werbebotschaften auf größere Aufmerksamkeit und Akzeptanz stoßen als isoliert und massiv ausgestrahlte Werbung. Vielleicht besteht das größte Wirkungspotenzial solcher Mischungen aus Informationsdiensten und Werbebotschaften darin, diesen Werbebotschaften trotz einer verbreiteten Abwehrhaltung gegenüber der Werbung im allgemeinen und der Werbung am POS im besonderen zu Akzeptanz und zu der angestrebten Wirkung zu verhelfen.

\section{c) Zu den Verhaltenswirkungen}

Auch die möglichen Verhaltenswirkungen von Informationsdiensten sind vielfältig. Hinweise auf den Lieferservice können Einkaufskörbe füllen und Kaufsummen steigern, Wetterhinweise ganz bestimmte Abverkäufe fördern, Produktinformationen die Markenwahl bestimmen und die Entlastung beim Einkaufen kann letztlich auch dazu beitragen, dass die Kaufzufriedenheit und damit auch die Geschäftstreue ansteigt.

\section{Zum Wirkungspotenzial des DS im Dienste der Ladenatmosphäre}

Bei der Skizze einiger Wirkungen des DS als atmosphärischer Faktor sei davon ausgegangen, dass es von seiner Auslegung her gesehen die Ladenästhetik, den Unterhaltungswert des Ladenbesuchs, die Orientierungsfreundlichkeit des Geschäfts und die soziale Dimension des Einkaufserlebens fördern soll. Des Weiteren wird davon ausgegangen, dass die Bildschirme verbreitet aufgestellt und die zentralen Inhalte über längere Zeit hinweg keine nennenswerten Veränderungen erfahren.

\section{a) Zu den Kontakterfolgen}

Beim skizzierten System, das DS als ,ambient factor“ gestaltet, ist davon auszugehen, dass viele, wiederholte Kontakte eher beiläufig und ohne großes Involvement erfolgen, aber dennoch wichtige Wirkungen entfalten.

\section{b) Zu den kognitiv-affektiven Wirkungen}

Das als „ambient factor“ ausgelegte DS verbessert die Ladenatmosphäre und damit das emotionale Shoppingerleben (Bagozzi et al. 1999; Mau 2009). Der Schwerpunkt der atmosphärischen Wirkungen kann unter anderem auf sensorischen Empfindungen beim Stöbern, Riechen und 
Probieren bzw. Schmecken liegen (Crader u. Zaichkowky 2008, S. 89-99). Über ein positives Laden- und Shoppingerleben beeinflusst DS dann auch die Wahrnehmung und Beurteilung der Angebote und die Wahrnehmung und Beurteilung medialer Botschaften. Die mit der angenehmen Ladenatmosphäre einhergehende positive Stimmungslage trägt dazu bei, dass Beurteilungen des Angebots positiver ausfallen als bei weniger guter Stimmung (zur Stimmungswirkung von Ladenumwelten siehe Donovan u. Rossiter 1982; Bost 1987, S. 30-56, 145-155; Silberer u. Jaekel 1996, S. 258-264). Das Laden- und Shoppingerleben beeinflusst des Weiteren die Wahrnehmung der Zeit und damit die wahrgenommene Aufenthaltsdauer. Eine angenehme Ladenatmosphäre verkürzt die empfundene Aufenthaltsdauer und dabei auch die empfundene Wartezeit an der Kasse. Wenn sich solche Effekte einstellen, wird letztlich auch die Zufriedenheit mit dem Geschäft und damit dessen Image positiv beeinflusst.

\section{c) Zu den Verhaltenswirkungen}

Eine Förderung des Laden- und Shoppingerlebens steigert nicht nur die Aufenthaltsdauer und die Kaufneigung, auch die Bereitschaft, ungeplante Käufe zu tätigen, sondern auch die Geschäftstreue und die Bereitschaft, das Geschäft weiter zu empfehlen und bei Gelegenheit andere Personen beim Einkaufen mitzunehmen bzw. zum Mitkommen einzuladen.

\section{Das Wirkungs- und Nutzenpotenzial im Funktionenmix}

Das soeben skizzierte Wirkungspotenzial betrifft DS-Anwendungen mit unterschiedlichen Schwerpunkten in Sachen Funktionalitäten. Dennoch sei festgehalten, dass die einzelnen Systeme immer eine breite Palette an Wirkungen entfalten können. Ein Sales Promotion-System kann auch die Ladenatmosphäre und das Image eines Geschäfts beeinflussen, ein auf Imageförderung ausgelegtes System auch Abverkäufe fördern und die Ladenatmosphäre verbessern und ein DS-System, das als ,ambient factor“ gestaltet wurde, kann dies ebenfalls.

Wenn bei einem DS-System keine Funktionalität eindeutig dominiert und stattdessen von vornherein ein Funktionenmix angestrebt wurde, steigt die Anzahl solch funktionenübergreifenden Wirkungen. Diese müssen dann aber keineswegs stärker ausfallen. Wenn die Ausprägung der einzelnen Funktion in solchen Systemen weniger stark ausfällt, dürfte sich damit deren spezifisches Wirkungspotenzial eher reduzieren.

\section{Wirkungen erster Anwendungen - Stand der Forschung}

In den vergangenen Jahren sind Studien durchgeführt worden, die wie die oben skizzierten Wirkungen ebenfalls
Hinweise auf das Wirkungspotenzial des DS liefern. Diese Hinweise sind einerseits viel konkreter als die theoretischen Skizzen, können die theoretischen Aussagen andererseits aber nur partiell abdecken. Über die bislang durchgeführten Studien und deren Befunde sei nun berichtet. Studien aus der kommerziellen Marktforschung, die nur in Teilergebnissen zugänglich sind, sollen dabei ebenso zur Sprache kommen wie die durchweg besser dokumentierten wissenschaftlichen Studien.

Bezogen auf die nordamerikanischen Analysen ist zu bedenken, dass erste DS-Anwendungen in Nordamerika früher installiert wurden als in Europa, so dass der Erfahrungshintergrund der Ladenbesucher dort ein vergleichsweise größerer ist.

\section{Registrierte Absatzerfolge}

Raymond Burke (2009) hatte die Gelegenheit, unveröffentlichte, meist kommerzielle nordamerikanische Studien zu sichten und deren Ergebnisse zu berichten. Diese Studien beziehen sich vornehmlich auf registrierte Absatzwirkungen der eingesetzten DS-Systeme und zeigen, so Burke (2009, S. 181-184), dass Ladenbesucher vor allem auf Neuigkeiten, Sonderangebote und saisonale Produkte und weniger auf traditionelle Markenwerbung reagieren. Die Absatzsteigerung falle bei umsatzstarken Artikeln zwar prozentual geringer, absolut gesehen dagegen stärker aus als bei umsatzschwachen, z. B. völlig neuen Produkten. Des Weiteren kann Burke (2009) berichten, dass die Beachtung von DS-Inhalten nicht nur mit der Tageszeit und dem Wochentag variiert, sondern auch mit den BildschirmStandorten und ihrer Reichweite. Außerdem zeige sich, dass kurze Nachrichten stärker wirken als längere und dass eine Produktwerbung nicht nur dem umworbenen Artikel, sondern auch der gesamten Produktgruppe zugute kommen kann.

Ergebnisse von Kundenbefragungen und -beobachtungen

Stadler (2006) untersuchte ein großformatiges Display im Hauptbahnhof Zürich sowie einen Verbund kleinformatiger Bildschirme in einem Schweizer Einkaufscenter. Ausgewertet wurden reichweitenbezogene Besucherfrequenzen und Leitfaden-Gespräche zum Thema Wirkungen und Wirkungsursachen. Der Bericht hält zwei zentrale Ergebnisse fest. Erstens: Die Bildschirme treffen auf eine generell hohe Akzeptanz. Und zweitens: Es sind in erster Linie die Bewegbilder, die (reflexartige) Aufmerksamkeit erzielen.

In einer Publitest-Studie (Publitest 2006) wurden 400 Besucher eines Schweizer Carrefour-Verbrauchermarktes beim Verlassen des Geschäfts zur Beachtung, zur Akzeptanz und $\mathrm{zu}$ ausgewählten Wirkungen des neuen Mediums „Flachbildschirme“ befragt. $74 \%$ der Befragten gaben 
an, dass ihnen die Bildschirme beim gerade erfolgten Besuch oder bei früheren Besuchen schon einmal aufgefallen seien. Bildschirme im Kassenbereich wurden öfter wahrgenommen als Bildschirme in den Verkaufszonen und diese wiederum öfter als Bildschirme im Eingangsbereich. Bezüglich der Erinnerung von Bildschirminhalten, bestehend aus Werbung und Nachrichten, wurde Werbung am häufigsten erinnert: $46 \%$ erinnerten sich an die Werbung, $23 \%$ an Carrefour-Aktionen, $24 \%$ an die Nachrichten und $18 \%$ an den Wetterbericht. Die besten Erinnerungswerte (spontane und gestützte Erinnerung) erzielten die Carrefour-Aktionen (39\%), gefolgt von der Werbung für Süßigkeiten/Snacks/Chips (24\%), Beauty/Hautpflege (24\%), Reinigungsmittel $(22 \%)$ und Automobile (21\%). Die Recognition Rate bei den 13 umworbenen Marken liegt bei $25 \%$. Pro Person wurden im Schnitt drei Marken wiedererkannt. Gute Werte wurden auch bei der Markenzuordnung erzielt, gemessen als Anteil jener Personen, die einem anonymisierten Produkt die richtige Marke zuordnen. Zur Wirkung der Werbung auf das Kaufverhalten werden folgende Angaben gemacht: $8 \%$ der Befragten sagten, sie hätten gezeigte Produkte schon häufig gekauft, $19 \%$ ab und zu, $16 \%$ selten und $55 \%$ nie (jeweils bezogen auf jene Besucher, die die Bildschirme schon einmal bemerkt haben).

In dieser Publitest-Studie (Publitest 2006) zeigte sich auch, dass das Geschäftsimage bzw. das Image von „Carrefour" durch das TV-System gefördert wird. Der Supermarkt wirkte bei den Befragten, die die Bildschirme schon einmal wahrgenommen hatten, mit den Screens moderner und attraktiver. Positiv ist auch die berichtete Akzeptanz: Die Mehrheit dieser Befragten bekundete, dass ihnen die Bildschirme gefallen: sehr gefallen sagten $38 \%$, ziemlich gefallen $40 \%$, weniger gefallen $12 \%$ und gar nicht gefallen $5 \%$. Rund zwei Drittel der Besucher vertraten die Auffassung, dass das Warten an der Kasse durch den TV-Einsatz ,auf angenehme Weise verkürzt“ werde.

Schnedlitz u. Berger (2005) untersuchten DS-Anwendungen in zwei Wiener Verbrauchermärkten: in dem einen Geschäft einen großen Bildschirm in der Obst- und Gemüseabteilung, auf dem Markenwerbung, Werbung für Angebot im Geschäft und „Kundeninformationen“ dargeboten wurden, in dem anderen Geschäft einen kleinen PC-Screen zwischen Kasse und Ausgang, dessen Inhalte zu 2/3 aus Nachrichten und zu 1/3 aus Werbung bestanden. In beiden Geschäften wurden insgesamt 593 Kunden mündlich befragt und 995 ausgewählte Kunden verdeckt beobachtet (S. 306 f.). Dem Bericht zufolge zeigen die Befragungsergebnisse, dass Männer die Bildschirme öfter bemerkten als Frauen und dass beide Anwendungen das Kaufverhalten gleich stark ,inspirierten“. Im Rahmen der Beobachtung konnte festgestellt werden, dass der kleine Bildschirm mehr Aufmerksamkeit erzielte. Diesen zunächst etwas überraschenden Befund erklären die Autoren damit, dass der kleine Bildschirm für die Studie neu aufgestellt wurde, während die andere Anwendung bereits zum Inventar gehörte.

Telschow u. Keller (2007) berichten von einer Studie, die in acht deutschen Lebensmittelmärkten durchgeführt wurde. In diesen Märkten waren versuchsweise PlasmaBildschirme installiert und mit einem Testprogramm bestückt, das aus eigens dafür produzierten Beiträgen, Tafeln und Werbespots bestand - aus professionell erstellten redaktionellen Beiträgen wie Wetter- und Sportmeldungen und aus Imagewerbung für das Handelsunternehmen. Die Inhalte wurden zu den Tageszeiten und Sortimenten passend ausgesteuert. Die Ergebnisse zeigen, dass die Akzeptanz und die Wirkungen solcher Systeme entscheidend von der Platzierung der Bildschirme und von den Inhalten abhängen. Immerhin konnte bei $42 \%$ der Besucher ,eine Aufmerksamkeit für das Medium" erreicht werden. Die Erinnerung an die Spots fiel recht unterschiedlich aus die Quote liegt zwischen 3\% und 26\%. Mehrverkäufe liegen zwischen $2 \%$ und $24 \%$ - Mehrverkäufe bei PreisPromotionen ausgenommen. Erfolgreiche Inhalte zeichnen sich durch einen Bezug zur aktuellen Einkaufssituation, durch eine Entlastung des Kunden und durch eine Zielgruppenaffinität bzw. eine regionale und zeitliche Aussteuerung aus. Aufmerksamkeit konnte vor allem durch die Kopplung von Bild und Ton, aber auch durch bewegte Bilder erreicht werden. Die Erinnerungsquote lag bei den Bewegbildern um $7 \%$ höher als bei Standbildern bzw. Anzeigen. Und eine Mischung aus 50\% Fremdwerbung, $20 \%$ Eigenwerbung und $30 \%$ Information und Unterhaltung wurde von über $90 \%$ der Befragten nicht als Werbung, sondern als „zusätzlicher Service“ empfunden.

Silberer u. Büttner (2008) untersuchten die Akzeptanz und Wirkungen sowie die Reichweite eines DS-Systems, das in zwei Lebensmittelgeschäften jeweils zwischen Kasse und Ausgang installiert war. Die Inhalte bestanden aus einer Mischung aus Werbung für das Geschäft, Werbung überregionale Markenhersteller, Werbung lokaler Einrichtungen und Nachrichten. Die Akzeptanz und die Wirkungen wurden per Befragung von insgesamt 347 Ladenbesuchern erhoben. $63 \%$ der Befragten waren Frauen. In Bezug auf die Akzeptanz des Ladenfernsehens im Allgemeinen und das DS im besuchten Geschäft ergab sich eine Art Indifferenz. Auf einer Skala von sehr gut bis sehr schlecht lag das durchschnittliche Urteil bei beiden Fragen nahe an dem Punkt, der mit ,weder gut noch schlecht" beschriftet war. Erfragt und überprüft wurden auch Werbewirkungen. Von den Personen, die zumindest schon einmal auf einen der Bildschirme geschaut hatten, es waren $70 \%$ aller Befragten, bekundeten wiederum $70 \%$, über das Laden-Fernsehen keine nützlichen Hinweise erhalten zu haben. $53 \%$ gaben an, auf Nachrichten zu achten, $11 \%$ gaben an, auf Werbung 
zu achten, und $36 \%$ gaben an, auf beides zu achten - auf Informationen und Nachrichten. Von den umworbenen Marken konnte pro Person im Schnitt eine Marke erinnert werden, bei ungestützter Erinnerung waren es im Schnitt nur 0,4 Marken.

In ihrer Besucherbeobachtung erfassten Silberer u. Büttner (2008) die faktischen Werbekontakte. Die an der Kasse anstehenden Besucher wurden in ihrer visuellen Zuwendung zu den beiden Bildschirmen verdeckt beobachtet. Die Beobachter hatten die Anzahl der Personen im vorgegebenen Radius und die Anzahl der Blickzuwendungen festzustellen und zudem die durchschnittliche Dauer der Blickzuwendung abzuschätzen. Diese Aufgabe bedurfte der sorgsamen Einübung. Jedenfalls wurde das Beobachtungsintervall auf fünf Minuten begrenzt und nach jedem Beobachtungsintervall eine Fünf-Minuten-Pause eingelegt, in der die Beobachtungsergebnisse dokumentiert werden konnten. Die Auswertung der Beobachtungsbögen ergab, dass sich im Schnitt zwölf Personen im Beobachtungsradius aufhielten, dass sich im Schnitt vier von ihnen mindestens einmal dem Bildschirm zuwandten und dass die geschätzte Betrachtungsdauer im Schnitt bei etwa vier Sekunden liegt.

Ergänzende Hinweise liefern zwei Studien, die in englischen Shopping Centern zum TV-Einsatz mit einem unterhaltenden TV-Programm und Werbeeinblendungen durchgeführt worden sind. Die eine Studie stammt von Newman et al. (2006), die andere von Dennis et al. (2008).

Newman et al. (2006) veranstalteten acht Gruppendiskussionen mit 51 Shopping Center-Besuchern, um auf diese Weise in Erfahrung zu bringen, wie der TV-Einsatz das Einkaufserleben und das Center-Image beeinflusst. $\mathrm{Zu}$ sehen waren Videos und Programme privater TV-Sender. Aus den Diskussionen ergaben sich den Autoren zufolge Hinweise darauf, dass die Wirkungen von den jeweiligen Standorten und den jeweiligen Personen abhängen. Einigkeit habe in der Auffassung bestanden, dass der TV-Einsatz einen atmosphärischen Faktor darstelle, der das Erleben des Shopping Center positiv beeinflusse, und dass auf diese Weise kaufrelevante Informationen vermittelt werden können.

Dennis et al. (2008) befragten Besucher eines Shopping Centers vor und nach der Einführung eines TV-Systems und zu gleicher Zeit die Besucher eines vergleichbaren Centers, in dem kein TV-System eingeführt wurde (insgesamt 389 Personen, z. T. postalisch, z. T. per Mail, z. T. mündlich/vor Ort). Anhand der quasi-experimentellen Untersuchungsanlage sollte in Erfahrung gebracht werden, inwieweit sich das Center-TV auf die Wahrnehmung und Beurteilung des Centers, auf das Einkaufserleben, die Aufenthaltsdauer und die getätigten Ausgaben auswirkt. Die Ergebnisse lassen Folgendes erkennen: Das Center-TV beeinflusst die Wahrnehmung des Centers und das Kaufverhalten - aber nur bei Berufstätigen, nicht bei Arbeitslosen und Rentnern. Es beeinflusst auch das Einkaufserleben und dieses wiederum das Kaufverhalten, so dass vom TV auch ein indirekter Einfluss auf das Kaufverhalten ausgeht.

Ergebnisse von Managerbefragungen

Um das Wirkungspotenzial von In-Store-Medien, zu denen auch das DS zu zählen ist, aus der Sicht von Führungskräften in Erfahrung zu bringen, wurden auch einige Managerbefragungen durchgeführt.

Sattler u. Hartmann (2004) führten in Deutschland Gespräche mit Experten aus Herstellerunternehmen, MediaAgenturen und POS-Media-Anbietern. Darüber hinaus erfolgte eine schriftliche Befragung von 88 „MarketingEntscheidern“, von denen $82 \%$ in der Konsumgüterindustrie arbeiteten, die übrigen in Media-Agenturen. Die befragten Experten bekannten, mit POS-Medien vor allem das Ziel der Abverkaufsförderung zu verfolgen. Eine Abverkaufsförderung sei zwar festzustellen, aber „nicht sehr beeindruckend". Nennenswerte Erfolge werden eher dem In-Store-Radio und den Floor Graphics zugeschrieben.

Eine IfH-Studie (2007) wandte sich an Führungskräfte mit der Bitte, den Stellenwert von „Medien am POS“ zu beschreiben. In einer Online-Umfrage wurden 550 Entscheider aus der Industrie, aus Werbe- und Media-Agenturen und aus Marketing- sowie Vermittlungs-Agenturen konsultiert. Dabei kam auch das DS zur Sprache, verstanden als ein Screen-System mit animierten Inhalten, mit Angebotshinweisen, Produktwerbung und Imagewerbung. Den Befragten zufolge komme DS zwar nicht flächendeckend im Einsatz, die Anzahl der Anwendungen sei aber stark wachsend. Die nicht näher beschriebene ,qualitative Kommunikationsleistung“ dieses Systems wird als mittelmäßig, die ebenfalls nicht näher beschriebene „quantitative Kommunikationsleistung" etwas besser eingestuft.

\section{Zusammenfassung der Befunde}

Angesichts der referierten, ersten Studien zu Wirkungen von DS-Systemen im stationären Handel lassen sich zusammenfassend folgende Befunde festhalten:

Die meisten im stationären Handel bislang installierten DS-Systeme dienen den befragten Führungskräften und Experten zufolge in erster Linie der Verkaufsförderung. Dabei kann das DS durchaus nennenswerte Kontakterfolge erzielen. Die faktischen Reichweiten differieren je nach Standort in Abhängigkeit vom Passantenaufkommen und von der Reichweite der Bildschirme. Eine relativ häufige und relativ lange Beachtung erfahren Bildschirme in der Kassenzone - verglichen mit der Beachtung in der Verkaufszone bzw. in der Präsentationsfläche. Eine besonders hohe Aufmerksamkeit erringen Bewegbilder, wobei es sich in erster Linie um eine reflexartige Zuwendung handelt. 
DS erzielt auch kognitiv-affektive Wirkungen. Bildschirme und deren Inhalte werden zumindest von einem Teil der Ladenbesucher wahrgenommen. Sie fallen in der Kassenzone stärker auf als im Verkaufs- bzw. Präsentationsbereich. Einige achten nur auf Werbung, andere nur auf Nachrichten, relativ viele auf beides. Unter den erinnerten Inhalten befinden sich auch Werbebotschaften und die beworbenen Marken. Der Recall von Werbebotschaften variiert mit der Gestaltung der Inhalte und mit den umworbenen Marken. DS beeinflusst auch die Ladenatmosphäre, fördert das Einkaufserleben und verkürzt die empfundene Wartezeit an der Kasse. Berichte deuten darauf hin, dass DS auch das Image des Händlers fördern kann. Ein Teil der Ladenbesucher geben auch an, dass ihnen die per DS vermittelten Inhalte bzw. die Screens gefallen hätten.

DS kann auch das Verhalten der Kunden beeinflussen. Registrierungen von Absatzerfolgen lassen erkennen, dass diese vor allem bei neuen Produkten, bei Sonderangeboten und bei saisonalen Angeboten erzielt werden können und dass sie mit dem Wochentag und der Tageszeit variieren. Sie fallen bei kurzen Werbespots stärker aus als bei längeren Werbebotschaften. Es zeigte sich auch, dass Absatzerfolge nicht nur dem beworbenen Produkt, sondern auch der ganzen Produktgruppe zugute kommen können. Die Angaben befragter Ladenbesuchern deuten eher auf begrenzte Kaufwirkungen hin, so dass zu vermuten ist, dass diese Wirkungen nicht immer bewusst wahrgenommen und damit etwas unterschätzt werden. Zu den erkennbaren Verhaltenswirkungen zählt auch die Verlängerung der Verweildauer und damit die gesteigerte Chance, Erinnerungskäufe und Impulskäufe auszulösen.

\section{Herausforderungen für und Empfehlungen an die Praxis}

Auch wenn die derzeit verfügbaren Erkenntnisse in Sachen DS im stationären Handel noch einige Lücken aufweisen, lassen sich doch bereits heute einige wichtige Empfehlungen an die Praxis formulieren. Diese beziehen sich zum einen auf die strategische Ausrichtung und zum anderen auf die konkrete Ausgestaltung eines DS-Systems.

Die strategischen Ausrichtung eines DS-Systems erfordert klare Entscheidungen und Vorgaben bezüglich seiner Funktionalität und damit auch zu seinem Beitrag zur Profilierung des Betriebes oder der Kette (zu Profilierungsstrategien im Einzelhandel siehe Liebmann et al. 2008, S. 148-200). Dabei sollte bedacht werden, dass sich mit einer DS-Anwendung auch andere Ziele als die Verkaufsförderung anstreben lassen. Vielleicht lohnt sich eine DS-Anwendung in vielen Fällen erst dann, wenn ihr mehrere Aufgaben übertragen werden. Als Kandidaten kommen dabei nicht nur die oben skizzierten Funktionalitäten in Frage (Imageförderung, Informationsdienste und Verbesserung der Ladenatmosphäre), sondern auch die Unterstützung der Werbewirkungsforschung sowie die verbesserte Corporate Identity der Mitarbeiter. Wenn die Sales Promotion derzeit als DS-Funktionalität dominiert, liegt dies vermutlich auch an dem Umstand, dass DS-Systeme oft von Dienstleistern vorfinanziert und über Werbeeinahmen aus der Industrie refinanziert werden müssen.

Die operative Ausgestaltung eines DS-Systems muss darauf achten, dass die richtigen Inhalte am richtigen Ort zur richtigen Zeit vorgehalten und dargeboten werden (,simple, direct, and relevant messages“ - so Burke 2009, S. 184). Dies ist genau so wichtig wie die Funktionstüchtigkeit von Hard-, Software- und Netzwerkkomponenten. Hervorgehoben sei auch die integrierte Kommunikation vor Ort sowie die Einbettung in das Marketing des Händlers am POS und in seinem Einzugsgebiet. Das Erreichen der gesteckten Ziele kann dann am ehesten gelingen, wenn immer wieder einmal Durchführungs-, Erfolgs- und Prämissenkontrollen durchgeführt werden. Die Durchführungskontrollen sollten sich nicht nur auf die technische Funktionstüchtigkeit beziehen (vielfach bleiben Bildschirme aus technischen Gründen einfach „schwarz“ oder „grau“), sondern auch auf die Gestaltung der Botschaften und das Content Management. Die Erfolgskontrollen können mit der Auswertung von Aufzeichnungen von Absatzerfolgen in den Scannerkassen beginnen, dürfen sich aber nicht darauf beschränken. Stichprobenartige Kontrollen von Kontakt-, Erlebens-, Einstellungs- und Verhaltenswirkungen müssen hinzukommen. Und bei den Prämissenkontrollen ist immer wieder einmal zu fragen, ob die anfangs zugrundegelegten Prämissen, z. B. die Annahmen bezüglich der Zielgruppen, der Nutzerschaft sowie der Kontakt-, Erlebens-, Einstellungsund Verhaltenswirkungen noch Gültigkeit beanspruchen können. Selbst wenn in Zukunft die DS-Systeme mit einer Lernfähigkeit ausgestattet werden und sich selbst steuern können, wird dies die Verantwortlichen nicht davon entbinden, regelmäßige Kontrollen durchzuführen und aus den Erfolgen bzw. Misserfolgen zu lernen.

\section{Leerfelder und Aufgaben für die künftige Forschung}

Während die künftige Praxis dazu beitragen kann, die Funktionalität von DS-Systemen zu erweitern und damit die Chance steigern kann, das Anwendungspotenzial einer DS-Technik konkreter zu beurteilen, sollte die künftige Forschung dazu beitragen, dass sich auch das Wirkungspotenzial von DS-Anwendungen besser beurteilen lässt. Dies wird umso mehr gelingen, je stärker sich die Forschung darum bemüht, das gesamte Wirkungsgeschehen bei DS-Anwendungen ins Blickfeld zu rücken. Dabei 
kann unterschieden werden zwischen einer Forschung, die sich auf einzelne Geschäftsbesuche bezieht, und einer Forschung, die sich für zeitübergreifende Wirkungen bei wiederholten Geschäftsbesuchen interessiert.

Auf einzelne Geschäftsbesuche bezogen zählen zu diesen Lücken vor allem folgende:

Erstens die nähere Beschreibung der DS-Systeme (Standorte, Inhalte, Timing), der Ladenbesucher (Interessen, Einstellungen, Gewohnheiten) und der Ladenumgebung (Sortimente, Präsentation, Mitarbeiter) als wichtige Determinantenbündel. Die Dokumentation dieser Bündel stellt eine Voraussetzung dafür dar, dass deren Einfluss auf das Wirkungspotenzial detaillierter und umfassender als bislang beurteilt werden kann.

Zweitens die gezielte Analyse von Wirkungen, die für einzelne Inhalte bzw. Content-Kategorien gelten, so z. B. die vergleichende Analyse von Wirkungen der Sortimentswerbung des Händler, der Markenwerbung der Hersteller, der Werbung regionaler Non-Profit-Organisationen, der Informationsangebote und der Serviceangebote.

Drittens die differenzierte Analyse des Wirkungsbündels und damit der selektiven Zuwendung zu einzelnen Bildschirmen, der Wahrnehmung einzelner Botschaften, ihrer Verarbeitung und Wirkungen sowie der Beurteilung des DS (Gefallen und Missfallen) und der atmosphärischen Effekte insgesamt.

Viertens die Versuche, das Ursache-Wirkungs-Geschehen näher $\mathrm{zu}$ bestimmen, nicht nur über das Erfragen wahrgenommener oder vermuteter Kausalitäten seitens der Kunden und der Experten, sondern auch über Experimente, die sich im Labor, aber auch im Feld durchführen lassen.

Bezogen auf DS-Systeme, die über längere Zeiträume hinweg eingesetzt werden, sind ebenfalls gravierende Lücken festzuhalten:

Erstens die Analyse von Änderungen der DS-Anwendungen im Zeitablauf, z. B. der Änderung von Standorten, der erweiterte Abdeckung von Geschäftsbereichen sowie der Änderung von Inhalten und ihrer Darbietung.

Zweitens die Analyse des Nutzerverhaltens im Zeitablauf, z. B. der veränderten Beachtung von Bildschirmen und einzelner Inhalte über längere Zeiträume hinweg. Dabei wäre es interessant zu erfahren, welche Änderungen sich aus der Gewöhnung heraus und welche sich aus positiven und negativen Erfahrungen heraus ergeben. Da nicht jede Neuerung dauerhafte Ablehnung oder dauerhafte Akzeptanz findet, ist auch für DS-Systeme nicht auszuschließen, dass sich eine anfänglich vorhandene Akzeptanz im Laufe der Zeit zurückbildet und in Ignoranz übergeht und dass nicht jede anfängliche Ablehnung auf Dauer bestehen bleibt.

Drittens die Analyse mittel- bis langfristiger Einstellungsund Verhaltenswirkungen, verzögerte Effekte einzelner Verkaufsförderungsaktionen inbegriffen (Gedenk 2002). Bei der Markenwerbung von Herstellern sollten dabei auch Kaufwirkungen außerhalb des jeweiligen Geschäfts interessieren.

Eine künftige Forschung, die solche Lücken schließen will, kann - wie bisher praktiziert - auf Registrierungen von Absatzerfolgen zurückgreifen, muss aber auch weiterhin Beobachtungs- und Befragungsstudien mit einschließen, auch das Tracking von Interessen, Einstellungen und Verhaltensweisen einzelner Ladenbesucher.

\section{Resumé}

Digital Signage steht für den vernetzten Einsatz von Flachbildschirmen als Informations- und Werbeplattform. Diese neue Plattform kommt im stationären Handel neuerdings verstärkt zum Einsatz. Dabei ist eine sehr enge Ausrichtung auf die Verkaufsförderung festzustellen. Das Anwendungspotenzial geht aber über die Verkaufsförderung weit hinaus und umfasst unter anderem die Imageförderung, den umfassenden Informationsservice und die Förderung der Ladenatmosphäre. Vor diesem Hintergrund und angesichts relevanter Wirkungstheorien sowie erster Studien lässt sich das Wirkungspotenzial des Digital Signage im stationären Handel durchaus abschätzen. Beim derzeitigen Wissensstand ist festzuhalten, dass Digital Signage im stationären Handel recht vielfältige Wirkungen entfalten kann, zu denen kognitiv-affektive Wirkungen wie Kenntnisse und Präferenzen ebenso zu zählen sind wie Wirkungen auf der Verhaltensebene, so z. B. die Verlängerung der Besuchsdauer sowie das Stimulieren ungeplanter Käufe. Zu den Erfolgsfaktoren zählen die Anzahl der Bildschirme, deren Reichweite, die Inhalte und deren Abstimmung auf die situative Verfasstheit des Ladenbesuchers am jeweiligen Standort sowie die Interessen und Gewohnheiten der Ladenbesucher. Für die Praxis gilt es künftig, die strategische Ausrichtung eines DS-Systems im Lichte der skizzierten Alternativen klar zu entscheiden und dann auch konsequent umzusetzen. Was die konkrete Ausgestaltung künftiger DS-Anwendungen betrifft, können bisherige und auch künftige Studien wichtige Anhaltspunkte liefern.

Open Access Dieser Artikel wird zu den Bedingungen der „Creative Commons Attribution Noncommercial License“ zur Verfügung gestellt. Damit ist eine nichtkommerzielle Nutzung, Verbreitung und Vervielfältigung erlaubt, sofern die Autoren des Artikels und die genaue Quelle angegeben sind.

\section{Literatur}

Ahlert D, Kenning P (2007) Handelsmarketing. Springer, Berlin Heidelberg New York

Bagozzi RP, Gopinath M, Nyer PU (1999) The role of emotions in marketing. J Acad Market Sci 27(2):184-206

Barth K, Hartmann M, Schröder H (2002) Betriebswirtschaftslehre des Handels, 5. Aufl. Gabler, Wiesbaden 
Bost E (1987) Ladenatmosphäre und Konsumentenverhalten. Physika, Heidelberg

Brown RG (1974) Sales response to promotions and advertising. J Advertising Res 14(49):33-39

Burke RR (2006) The third wave of marketing intelligence. In: Krafft M, Mantrala M (Hrsg) Retailing in the 21th century. Current and future trends. Springer, Berlin

Burke RR (2009) Behavioral effects of digital signage. J Advertising Res 49(2):180-185

Büttner O (2009) Kognitive Prozesse am Point of Sale. Zur Qualität von Datenerhebungsmethoden der Konsumentenforschung. Gabler Edition Wissenschaft (Interaktives Marketing). Gabler, Wiesbaden

Chandon P, Hutchinson JW, Bradlow E, Young SH (2009) Does instore marketing work? Effects of the number and position of shelf facings on brand attention and evaluation at the point of purchase. J Marketing 73(6):1-12

Clark D (2009) Framing effects exposed. Pearson Education, New York

Constantinides E, Romero CL, Gomez Boria MA (2008) Social media: A new frontier for retailers? Eur Retail Res 22:1-28

Crader S, Zaichkowsky JL (2008) The art of marketing. In: Lowrey TM (Hrsg) Brick and mortar shopping in the 21th century. Lawrence Erlbaum, New York London

Dennis C, Michon R, Newman A (2008) The impact of Digital Signage as a retail atmospheric tool. Paper presented at the Annual Conference of the Academy of Marketing Science, Vancouver, BC, Canada, May 28-31, 2008

Dhar R, Huber J, Khan U (2007) The shopping momentum effect. J Marketing Res 44(3):370-378

Diller H (1999) Discounting: Erfolgsgeschichte oder Irrweg. In: Beisheim O (Hrsg) Distribution im Aufbruch. Vahlen, München

Donovan RJ, Rossiter JR (1982) Store atmosphere - an environmental psychology approach. J Retailing 58(1):34-57

Druckman J (2001) Evaluating framing effects. J Econ Psychol 22(1): 96-101

EHI (2005) Visionen im Handelsmarketing. EHI Retail Institute, Köln

Fischer L (2002) Kiosksysteme im Handel. Einsatz, Akzeptanz und Wirkungen. Gabler Edition Wissenschaft (Interaktives Marketing). Gabler, Wiesbaden

Foscht T, Angerer T (2006) Handelswerbung - Strategien und Instrumente. In: Zentes J (Hrsg) Handbuch Handel. Gabler, Wiesbaden

Fritz W, Lorenz B, Hauser U (2007) Die Discountisierung der Gesellschaft. Deutscher Betriebswirte-Verlag, Gernsbach

Gedenk K (2002) Verkaufsförderung. Vahlen, München

Gerba B (2009) The 2009 Digital Signage study: Costs have fallen $23 \%$. http://www.wirespring.com. Letzter Zugriff: 22.11 .2009

Gröppel A (1991) Erlebnisstrategien im Einzelhandel. Physika, Heidelberg

Gröppel-Klein A (2006) Point-of-Sale-Marketing. In: Zentes J (Hrsg) Handbuch Handel. Gabler, Wiesbaden

Gröppel-Klein A, Bartmann B (2009) Turning bias and walking patterns: Consumers' orientation in a discount store. Marketing JRM 29(1):43-56

Hawkins SA, Hoch SJ, Meyers-Levy J (2001) Low-involvement learning: Repetition and coherence in familiarity and belief. J Consum Psychol 11(1):1-11

IfH (2007) Status-Quo: at-retail-media. Eine Befragung zur Verwendung und Bewertung von Medien am POS. Institut für Handelsforschung (IfH), Köln

Jacobsen M (1994) Kunst im Unternehmen. Campus, Frankfurt/M. New York

Karremans JC, Stroebe W, Claus J (2006) Beyond Vicary's fantasies: The impact of subliminal priming and brand choice. J Exp Soc Psychol 42(6):792-798
Kozinets RV (2008) Brands in space: New thinking about experiential retail. In: Lowrey TM (Hrsg) Brick and mortar shopping in the 21th century. Lawrence Erlbaum, New York London

Kroeber-Riel W (1993) Bildkommunikation. Vahlen, München

Kroeber-Riel W, Weinberg P, Gröppel-Klein A (2009) Konsumentenverhalten, 9. Aufl. Vahlen, München

Krugman HE (1965) The impact of television advertising: Learning without involvement. Public Opin Quart 29(3):349-356

Lademann RP (1999) Verkaufsflächenexpansion als Herausforderung der Stadtplanung. In: Beisheim O (Hrsg) Distribution im Aufbruch. Vahlen, München

Liebmann H-P, Zentes J, Swoboda B (2008) Handelsmanagement, 2. Aufl. Vahlen, München

Mattmüller R (1999) Image-Malus des Handels und rechtliche Konsequenzen. In: Beisheim O (Hrsg) Distribution im Aufbruch. Vahlen, München

Mau G (2009) Die Bedeutung der Emotionen beim Besuch von OnlineShops. Gabler, Wiesbaden

Morschett D (2006) Retail-Branding - Strategischer Rahmen für das Handelsmarketing. In: Zentes J (Hrsg) Handbuch Handel. Gabler, Wiesbaden

Newman A, Dennis C, Zaman S (2006) Marketing images and consumers' experiences in selling environment. Market Manage J 2006/Fall:1-17

Novak TP, Hoffman DL (2009) The fit of thinking style and situations: New measures of situations-specific experiential and rational cognition. J Consum Res 36(1):56-72

OgilvyAction (2007) Shopper decisions made in-store. OgilvyAction, New York

Petty RE, Cacioppo JT (1984) The effects of involvement on responses to argument quantity and quality: Central and peripheral routes to persuasion. J Pers Soc Psychol 46(1):69-81

Petty RE, Cacioppo JT (1986) The elaboration likelihood model of persuasion. In: Berkowitz L (Hrsg) Advances in experimental social psychology. Academic Press, New York

Publitest AG (2006) Neo Advertising. Medienwirkungsforschung „Carrefour Dietlikon“. Publitest AG, Zürich

Riediger P, Röder A (2007) Maßgeschneidert passt es besser. Lebensmittel Zeitung vom 21.9.2007, S 50

Rogers EM (2003) Diffusion of innovations, 5. Aufl. Free Press, New York

Rudolph T (2005) Modernes Handelsmanagement. Pearson Studium, München

Sattler H, Hartmann A (2004) POS-Marketing. Instore Medien aus Sicht der Marketing-Entscheider. absatzwirtschaft 2:11-15

Schnedlitz P, Berger D (2005) Die Wirkung von Info-Bildschirmen am POS - Ausgewählte Befunde einer empirischen Untersuchung im LEH. In: Trommsdorff V (Hrsg) Handelsforschung 2005. Kohlhammer, Stuttgart

Schüppenhauer A (2000) Trends, Szenen, Mythen und Kult - Das Marketing von Alltags-Sinn. In: Foscht T, Jungwirth G, Schnedlitz P (Hrsg) Zukunftsperspektiven für das Handelsmanagement. Deutscher Fachverlag, Frankfurt/M.

Schwarz N (2002) Situated cognition and th wisdom of feelings: Cognitive tuning. In: Feldman Barret L, Salovey P (Hrsg) The wisdom in feeling. Gilford Press, New York

Schweiger G, Schrattenecker G (2009) Werbung, 7. Aufl. Lucius \& Lucius, Stuttgart

Silberer G (1999) Handelsmarketing mit neuen Medien. Herausforderungen für den klassischen und den elektronischen Handel. In: Beisheim O (Hrsg) Distribution im Aufbruch. Bestandsaufnahme und Perspektiven. Vahlen, München

Silberer G (2000) Der Einsatz von Kiosksystemen als Werbeträger. In: Silberer G, Fischer L (Hrsg) Multimediale Kioskterminals. Infotankstellen, Telekommunikationsstellen und Smart Shops der Zukunft. Gabler, Wiesbaden 
Silberer G (2009) Digital Signage in der Kassenzone. POS Manager Technol 2:62-63

Silberer G, Büttner O (2008) Kontaktchancen, Kontaktqualität und Werbewirkungen der Kommunikations-Plattform Store-TV. Ergebnisse einer Feldstudie. Institut für Marketing und Handel, Universität Göttingen

Silberer G, Engelhardt J-F, Volland M (2004) POS-Terminals als Orientierungshilfe für den Ladenbesucher. der markt 43(169):82-88

Silberer G, Fischer L (2000) Akzeptanz, Wirkungen und Erfolge von Kiosksystemen. In: Silberer G, Fischer L (Hrsg) Multimediale Kioskterminals. Infotankstellen, Telekommunikationspunkte und Smart Shops der Zukunft. Gabler, Wiesbaden

Silberer G, Jaekel M (1996) Marketingfaktor Stimmungen. SchäfferPoeschel, Stuttgart

Silberer G, Köpler B-H, Marquardt J (2000) Kommunikation mit Kunst im Unternehmen. Deutscher Fachverlag, Frankfurt/M.

Smith ER, Semin GR (2004) Socially situated cognition: Cognition in its social context. Adv Exp Soc Psychol 36:53-117

Sörensen H (2009) The in-store ,audience“. J Advertising Res 49(2): $176-179$

Stadler U (2006) Out-of-Home-Displays wirkungsvoll einsetzen. Marketing Kommunikation 1:61-63

Stottmeister G (1990) Werbewirkung der Shopping-Center-Plakatierung. Dynamik Handel 35(6):11-15

Swoboda B (1996) Interaktive Medien am Point of Sale: Verhaltenswissenschaftliche Analyse der Wirkungen multimedialer Systeme. Gabler, Wiesbaden
Swoboda B (1998) Conditions of consumer information seeking: Theoretical foundations and empirical results of using interactive multimedia systems. Int Rev Retail Distrib Consum Res 8(4):361381

Telschow S, Keller F (2007) Inhalte: Schlüssel zum Erfolg, retail technology/spezial 2007:40-41

Titus PA, Everett PB (1995) The consumer retail search process: A conceptual model and research agenda. J Acad Market Sci 23(2):106-119

Todd A (2009) The Digital Signage revolution. http://revistaminimi. com. Letzter Zugriff: 22.11.2009

Tversky A, Kahneman D (1981) The framing of decisions. McGrawHill, New York

Van Loock N, Vermeir I, Geuens M (2009) The effect of advertising complexity and repetition on attitude toward digital signage advertisements. University College Ghent, Faculty of Business Administration and Public Administration

Walsh G (2002) Konsumentenverwirrtheit als Marketingherausforderung. DUV/Gabler, Wiesbaden

Wang K-Y, Peracchio LA, Luna D (2008) The role of thin slice judgments in retail environments. In: Lowrey TM (Hrsg) Brick and mortar shopping in the 21th century. Lawrence Erlbaum, New York London

Woodside AG, Waddle GL (1975) Sales effects of in-store advertising. J Advertising Res 15(3):29-33

Zielke S (2002) Kundenorientierte Warenplatzierung. Modelle und Methoden für das Category Management. Kohlhammer, Stuttgart 